\title{
EUROGEO: Una asociación europea enfocada a la investigación y a impulsar una docencia innovadora, que hoy marca el futuro de la Geografía en Europa
}

\section{INTRODUCCIÓN}

EUROGEO es una asociación internacional sin ánimo de lucro con sede en Bélgica, denominada European Association of Geographers (Asociación Europea de Geógrafos). Es la heredera de la European Standing Conference of Geography Teachers' Associations (ESCGTA), que surge en el seno de la UE, entonces EEC, financiada por la Comisión Europea. Inicialmente aglutina asociaciones que impulsan la enseñanza de la Geografía en Europa. Este hecho hace que pase a llamarse European Network of Geography Teachers' Associations (EUROGEO), y a considerarse una red.

Sin embargo EUROGEO hoy no es una asociación de asociaciones, como antaño, sino que es una asociación que acoge personas o instituciones con un interés demostrado por la Geografía, su teoría, métodos y práctica. Por tanto, la adscripción a la asociación hoy es personal, si bien, existe la posibilidad de que las asociaciones formen parte de ella, como se explicita claramente en sus estatutos. Por tanto existen dos formas de asociarse, una individual y otra como asociación. Las adscripciones de asociaciones cuentan con un tratamiento distinto al de la adscripción personal, según los estatutos de la Asociación.

A partir del año 1994 la asociación se amplía para acoger no sólo a los países de la UE, sino a los de toda Europa, siendo entonces cuando toma el nombre de EUROGEO, por el que hoy la conocemos. Este era el nombre del Boletín de Geografía que se publica en el seno de la Asociación desde 1980 (figura 1). 
FIGURA 1

PORTADA DEL EUROGEO. BOLETÍN DE GEOGRAFÍA (NÚMERO 4)

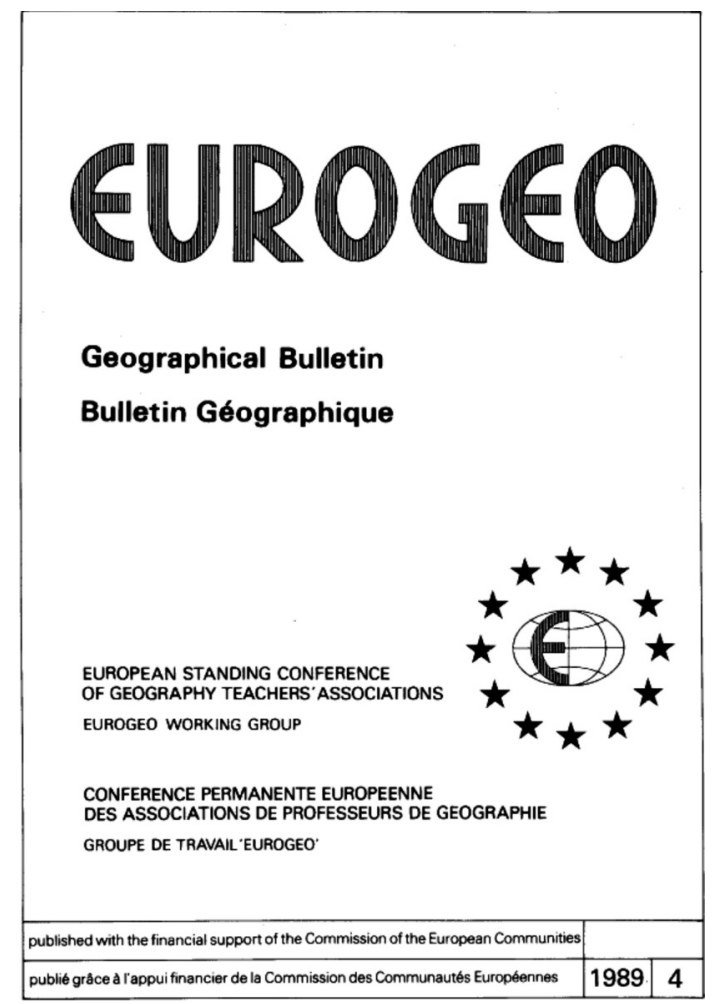

Desde sus inicios han participado en ella distintas asociaciones españolas, como la Real Sociedad Geográfica con una continuidad de más de tres décadas en la Asociación, y el Grupo de Didáctica de la Geografía, de la Asociación de Geógrafos Españoles, por su obvia afinidad de intereses. En el año 2004 la profesora Riera aconseja a la Asociación de Geógrafos Españoles su incorporación a EUROGEO.

En las páginas siguientes desarrollaremos el origen y la evolución de la Asociación, su continuado trabajo en los treinta y cinco años de su existencia, hasta llegar al relevante papel que hoy ocupa en la Geografía en Europa y su importancia en el futuro de la misma. También se aborda la participación de las asociaciones geográficas españolas en EUROGEO. 


\section{LOS PRIMEROS AÑOS DE EUROGEO}

EUROGEO surge como Asociación de asociaciones en el año 1979, en el seno de la CEE (hoy UE). Uno de sus principales impulsores desde el origen fue el profesor Henk Meijer, de la Universidad de Utrecht, aunque existen muchos nombres de geógrafos relevantes que tuvieron un importante papel en los inicios de la misma, entre otros: Andrew Convey (Universidad de Leeds), Hugo Heim (Universidad de Basel), Kirsten Herbard-Jørgensen (Universidad de Roskilde), Jean-Pierre Vandenbosch (Universidad de Bruxelles), José Estébanez (Universidad Complutense de Madrid).

Desde 1980 sus actividades principales consisten en una Conferencia Plenaria, que se celebra cada dos años en Bruselas, y un Boletín que se publica entre conferencia y conferencia gracias a la asistencia financiera de la Comisión y del Consejo de Europa (tabla 1).

TABLA 1

CONFERENCIAS DE LA ESCGTA, DESDE 1994 LLAMADA EUROGEO

\begin{tabular}{lc|ccc}
\hline \multicolumn{2}{c|}{ Conferencias celebradas } & \multicolumn{3}{c}{ Boletines } \\
\hline Año & Lugar & Año & N $^{\text {o }}$ & Tema \\
\hline 1980 & Bruselas (Bélgica) & 1981 & 0 & Migraciones \\
1982 & Bruselas (Bélgica) & 1983 & 1 & Turismo \\
1984 & París & 1985 & 2 & Problemas Regionales \\
1986 & Bruselas (Bélgica) & 1987 & 3 & Industria \\
1988 & Bruselas (Bélgica) & 1989 & 4 & Energia y Medioambiente \\
1990 & Bruselas (Bélgica) & 1991 & 5 & Agricultura \\
1992 & Bruselas (Bélgica) & 1993 & 6 & Tráfico y transporte \\
1994 & Bruselas (Bélgica) & 1995 & 7 & Demografía y Migraciones \\
1996 & Salzburgo & 1997 & 8 & Aspectos destacados de los distin- \\
\hline
\end{tabular}

Fuente: María Luisa Lázaro y Torres ${ }^{1}$.

${ }^{1}$ Más información en el informe realizado por la autora en la página Web de la Real Sociedad Geográfica (http://www.realsociedadgeografica.com/es/site/eurogeo.asp). 
La información aportada en los boletines estaba directamente relacionada con la dimensión europea de la enseñanza de la Geografía, lo que resultaba de gran utilidad para el profesorado en unos años en los que el acceso a la información tenía grandes dificultades. Se aportaban materiales para trabajar los hechos más relevantes de los países europeos. Se cuidaba especialmente el material gráfico, con la idea de que la lengua no constituyera una barrera. La primera parte contenía mapas mudos, temáticos, tablas estadísticas, gráficos... que tratan en conjunto a todos los países de la UE. En la segunda parte del Boletín se tratan aspectos geográficos concretos de la realidad de los países de las asociaciones participantes, siempre con una cuidada calidad, de forma accesible y sintética. Inicialmente se toma un tema transversal para todos los países, como se puede apreciar en la tabla 1, salvo en el último número, en el que será un aspecto a elegir libremente por cada país, en el caso de la aportación española, realizada por la autora de estas líneas, versó sobre el turismo. La repercusión de la importancia de estos boletines fue grande, como demuestra el hecho de que el primero de ellos está citado en la Red del Conocimiento o Web of Knowledge (WOK) ${ }^{2}$ en una reseña realizada en la revista Geography.

La importancia alcanzada por la asociación hizo que en 1989 se convirtiera en órgano consultivo del Consejo de Europa, privilegio con el que cuentan muy pocas asociaciones europeas y del que sigue disfrutando hoy en día. Años después, el cambio en las políticas comunitarias dejó sin financiación a la Asociación, y por tanto, se dejaron de publicar los boletines a finales de los noventa.

\section{Karl Donert, Presidente}

Cuando la Comisión Europea deja de financiar EUROGEO, surge una persona que será pieza clave dentro de la Asociación, un formador de profesores de la Universidad de Liverpool que obtuvo la mención de «International Fellow» en el Reino Unido. Ha escrito multitud de libros y liderado proyectos relacionados con el uso de las tecnologías encaminadas a la mejora de la enseñanza y el aprendizaje de la Geografía. Uno de los proyectos que en su momento coordinó, el proyecto europeo Minerva EURO.GEO, permitió

2 EUROGEO - GEOGRAPHICAL BULLETIN, 1981 O - STANDING-C-EUROPEAN-GEOGR-TEACHERS-ASS. Author(s): MINSHULL, GN (MINSHULL, GN). Source: GEOGRAPHY, Volume: 67, Issue: 296, Pages: 271-272, Published: 1982. Accession Number: WOS: A1982NY02100012. Publisher: GEOGRAPHICAL ASSN, 343 FULWOOD ROAD, SHEFFIELD, N YORKSHIRE, ENGLAND S10 3BP. IDS Number: NY02. ISSN: 0016-7487. 
organizar la conferencia anual de 2001, que sigue a la celebrada en Luxemburgo en 1998. El proyecto EURO.GEO, estrechamente relacionado con los fines y objetivos de EUROGEO, estaba centrado en promocionar la ciudadanía y la dimensión europea a través de la enseñanza de la Geografía, creando materiales, evidencias, consejos, etc. y nuevas oportunidades en la Red para crear y evaluar los recursos geográficos «on line». Esta conferencia de 2001 puede considerarse un hito en la historia de la Asociación, ya que uno de sus resultados principales se materializó en solicitar en 2002 la red HERODOT, dedicada al impulso de la Geografía en la enseñanza superior, en el marco del proceso de Bolonia. Está coordinada desde sus inicios por K. Donert desde la Universidad de Liverpool y financiada por la Comisión Europea. Desde entonces, miles de personas han trabajado en los eventos impulsados por el profesor Karl Donert y organizados por la asociación EUROGEO, favoreciendo así el trabajo en red entre diversas asociaciones e instituciones europeas, fomentando foros de intercambio de experiencias y difundiendo buenas prácticas docentes, profesionales e investigadoras en Europa, no sólo entre docentes e investigadores, sino entre los jóvenes geógrafos.

\section{HERODOT Y EUROGEO}

La red temática HERODOT o European Network of Geography in Higher Education, tendrá dos fases, una primera de 2002 a 2005, en la que comienza con unas 40 organizaciones, entre las que podemos señalar a la Universidad Autónoma de Barcelona y la de Madrid; y una segunda, para la que se consiguió financiación de la Comisión Europea para desarrollar algunos aspectos iniciados en la primera, conocida como HERODOT II. Su duración fue de 2005 a 2009 y llegará a unas 200 organizaciones europeas, de las que una docena son instituciones españolas. Uno de los principales resultados de esta segunda fase fue impulsar la Asociación EUROGEO.

La conferencia anual organizada por la asociación portuguesa APG en Madeira en el año 2002, establece el Presidium o Junta Directiva de la Asociación, con Karl Donert como presidente, y se comienza la estabilización y crecimiento de EUROGEO. En estos años, además de las conferencias anuales organizadas por la misma, la Asociación participa en muchas otras conferencias, entre las que podemos destacar las dos conferencias de Londres (2003 y 2006). La primera de ellas fue la conferencia de puesta en marcha de la red HERODOT, organizada por la Real Sociedad Geográfica británica con el Instituto de Geógrafos Británicos. La segunda trató sobre la innovación en el currículum de 
Geografía y su organización estuvo a cargo del Instituto de Educación de la Universidad de Londres. También se celebraron algunas otras conferencias en relación con las TIC y la Geografía, como la organizada por la Universidad de Brno (República Checa, 2005) y la conferencia sobre Geografía, Culturas y Civilización organizada por la UGI en Roma (Italia. 2005), entre otras.

La red temática, en su segunda fase, realiza un importante trabajo a través de los objetivos principales de promoción de la ciencia geográfica y su contribución a la mejora de la enseñanza de la Geografía mediante la mejora de las competencias del profesorado y el impulso a la profesionalización del geógrafo que se plasman en cuatro acciones principales (2006-2010):

1. La dimensión europea de la geografía en la enseñanza superior: ciudadanía, cultura, lugar e identidad.

2. La promoción de la geografía en el siglo Xxi y sus aportaciones a la sociedad.

3. La enseñanza innovadora de la geografía en Europa y acerca de Europa.

4. «Lifelong learning», estrategias de aprendizaje encaminado a la competitividad, obtención de empleo, inclusión social, etc.

Karl Donert logra así, a través de la única red sobre Geografía en Europa, la financiación necesaria para dar un nuevo impulso a EUROGEO convocando, de forma conjunta, las conferencias anuales propias de la Asociación (tabla $\mathrm{n}^{\circ} 2$ ), en el seno de las cuales se celebran las asambleas anuales de la Asociación, en las que se presentan el informe anual, generalmente también disponible en Internet y la renovación cada dos años del Presidium de la misma. En estos años se ha incrementado el número de conferencias en las que participa la Asociación, lo que denota un importante crecimiento y desarrollo. Podemos señalar las que organizó junto con $\mathrm{EUGEO}^{3}$ y la Universidad de Amsterdam (Países Bajos, 2007) sobre Geografía de Europa, y la participación de la Asociación en todas las conferencias de EUGEO. También participa con su propio «track» en las conferencias de la Unión Geográfica Internacional (UGI), la última de ellas, organizada por la Universidad de Colonia (Alemania). En ella organizó además un homenaje al profesor Hartwig Haubrich de la Universidad de Friburgo, autor y promotor desde la Comisión de Educación Geográfica de la UGI de las Declaraciones Internacionales sobre la Educación Geográfica (1992), la diversidad cultural (2000) y la Declaración de Lucerna sobre Educación Geográfica para el Desarrollo Sostenible (2007).

\footnotetext{
${ }^{3}$ Más información sobre EUGEO en http://www.realsociedadgeografica.com/es/site/eugeo.asp
} 
Algunos miembros de EUROGEO forman parte de la Comisión de Educación Geográfica de la UGI, entre ellos cabe destacar a la Vicepresidenta de la Asociación, Daniela Schmeinck.

Uno de los primeros cambios que sufre EUROGEO, en estos años, se deriva del hecho de que los geógrafos europeos no se dedican únicamente al

TABLA 2

CONFERENCIAS DE EUROGEO

\begin{tabular}{|c|c|c|c|}
\hline Año & Lugar & $\begin{array}{l}\text { Institución que comparte } \\
\text { la organización }\end{array}$ & Tema \\
\hline 1998 & Luxemburgo & $\begin{array}{l}\text { Luxembourg Geography } \\
\text { Association }\end{array}$ & Geography in Europe \\
\hline 2001 & Liverpool (Reino Unido) & Universidad de Hope & $\begin{array}{l}\text { Teaching geography, skills } \\
\text { and curricula }\end{array}$ \\
\hline 2002 & $\begin{array}{l}\text { Funchal (Madeira, Por- } \\
\text { tugal) }\end{array}$ & $\begin{array}{l}\text { APG, Associação de Professores } \\
\text { de Geografia }\end{array}$ & $\begin{array}{l}\text { Geography teaching in an enlar- } \\
\text { ging Europe }\end{array}$ \\
\hline 2004 & Bled (Eslovenia) & $\begin{array}{l}\text { University of Ljubljana and the } \\
\text { Slovenian Geography Teacher } \\
\text { Association }\end{array}$ & New developments in Geography \\
\hline 2005 & Torú́ (Polonia) & $\begin{array}{l}\text { Institute of Geography, } \\
\text { Nicolaus Copernicus University }\end{array}$ & $\begin{array}{l}\text { Changing Horizons in Geography } \\
\text { Education }\end{array}$ \\
\hline 2007 & Estocolmo (Suecia) & Stockolm University & $\begin{array}{l}\text { Geography for Society: Putting } \\
\text { Bologna into Action }\end{array}$ \\
\hline 2008 & Liverpool (Reino Unido) & Liverpool Hope University & Future Prospects in Geography \\
\hline 2009 & $\begin{array}{l}\text { Ayvalik } \\
\text { (Balikesir, Turkey) }\end{array}$ & Balikesir University & Celebrating Geographical Diversity \\
\hline 2010 & Praga (República Checa) & Charles University & Sustainable Geographies \\
\hline 2011 & Atenas (Grecia) & $\begin{array}{l}\text { Laboratory of Geography of the } \\
\text { National Technical University of } \\
\text { Athens, Grece, NTUA }\end{array}$ & $\begin{array}{l}\text { Geography: Your world - } \\
\text { A European Perspective }\end{array}$ \\
\hline 2012 & Dublín (Irlanda) & St. Patrick's University College & $\begin{array}{l}\text { Geography and Global Understan- } \\
\text { ding: Connecting the Sciences }\end{array}$ \\
\hline 2013 & Brujas (Bélgica) & Universidad de Gante & $\begin{array}{l}\text { Geography: Linking Tradition } \\
\text { and Future }\end{array}$ \\
\hline 2014 & La Valleta (Malta) & Universidad de Malta & $\begin{array}{l}\text { The Power of Geography and } \\
\text { the Role of Spatial Information }\end{array}$ \\
\hline
\end{tabular}

Fuente: María Luisa Lázaro y Torres, Karl Donert y web de EUROGEO. 
campo de la educación y de la enseñanza, sino que también se dedican a la investigación y a otros campos profesionales. De esta forma se plantea, durante la conferencia anual en Liverpool (2008), una Asociación que no sólo se centre en temas educativos, sino también en temas científicos, aplicados y en investigación. La conferencia de Ayvalik (Turquía, 2009) mostrará una asociación fuerte y reorganizada.

El continuo incremento en el número de asistentes y de nacionalidades distintas, hasta rondar las 40 nacionalidades diferentes en la conferencia celebrada en Brujas, ha seguido creciendo cada año hasta el punto de que se prevé que ronde las 200 personas en la próxima conferencia que se celebrará en la Universidad de Malta del 15 al 19 de mayo de $2014^{4}$.

En los últimos años las conferencias de EUROGEO, en sesiones paralelas, han acogido a la Asociación Europea de Estudiantes de Geografía: EGEA (European Geography Student Association), en la que cada vez tiene más peso la participación española.

\section{DigITAL-EARTH.EU Y EUROGEO}

A la red HERODOT II, seguirá la red digital-earth.eu: geomedia in schools (d-e.eu $)^{5}$ (510010-LLP-1-2010-1-AT-COMENIUS-CNW), financiada por la Comisión Europea a través del Programa Lifelong Learning (Comenius, UE) y liderada por el Zentrum für Geoinformatics (Z_GIS) de la Universidad de Salzburgo Paris Lodron, con una duración de tres años (2010-2013) y una participación de más de 50 instituciones interesadas por la Geografía, entre ellas la Real Sociedad Geográfica, la Universidad Complutense de Madrid, el Grupo de Didáctica de la Geografía (AGE), la Universidad de León, el IES San Roque de Badajoz...

El objetivo del proyecto es impulsar la utilización de los medios tecnológicos digitales en el aprendizaje de la Geografía, lo que se ha denominado «geo-media» (abreviatura de geographic media), entendiendo por tal la visualización de la información geográfica (geodatos, imágenes...) desde distintos

\footnotetext{
${ }^{4}$ La información sobre el evento está disponible en la página web de la asociación http://www.eurogeography.eu/conference/2014-malta/malta2014.html.

${ }^{5}$ Una amplia información sobre esta red se puede consultar en Buzo Sánchez, I. (2011): "Red Europea Comenius digital-earth.eu". Didáctica Geográfica, 12, pp. 157-161. Disponible en; http://www.didacticageografica.es/index.php/didacticageografica/article/view/67/67 (Fecha de consulta: 01/08/2013).
} 
medios de comunicación digital que la Web 2.0, los dispositivos móviles y los ordenadores favorecen. Supone la existencia de un contenido digital de calidad, relacionado con el espacio geográfico, es decir, geolocalizado de alguna manera, como pueden ser los contenidos procedentes de los globos virtuales, los geovisores, SIGWeb, la teledetección, Sistemas de Información Geográfica... Esta información geográfica o geoinformación ${ }^{6}$ facilita una aproximación a la enseñanza-aprendizaje desde lo local a lo global. Con ello se pretende colaborar con la Agenda Digital Europea 2020 en las escuelas del siglo XXI.

La Red se organiza en cuatro grupos de trabajo, cada uno especializado en alguno de los aspectos considerados fundamentales para impulsar el aprendizaje de la Geografía: 1. Recursos, tecnologías y geoinformación; 2. Aprender y enseñar con los «geo-media» y la geoinformación; 3. Formación del profesorado y capacitación en «geo-media»; y 4. Aspectos curriculares.

En el seno del proyecto se impulsa la formación del profesorado a través de la creación de centros de innovación en Europa con la calificación de «Centros de Excelencia Europeos». Todos ellos deberán reunir unos estándares acordados y elaborados por un Centro de Excelencia Europeo. Será de nuevo Karl Donert, como experto en redes, el que cree y dirija el Centro de Excelencia Europeo con sede en la Universidad Paris-Londron de Salzburgo (Austria, 2011). Su finalidad será la misma que la de la Red, promover el uso de los «geo-media» y de la información espacial en los centros educativos y en la formación de profesores, para mejorar el pensamiento espacial y el aprendizaje de la Geografía. El centro crea e impulsa una red europea de centros de excelencia, en continuo crecimiento. En la actualidad cuenta con $20 \mathrm{CoE}$ (Centers of Excellence). El objetivo final de estos centros es impulsar una geografía innovadora que aproveche los medios tecnológicos y digitales «geo-media» en la investigación, difusión y el aprendizaje de la Geografía y colaborar así con la Agenda Digital Europea 2020.

La Real Sociedad Geográfica lidera uno de estos centros, denominado Real Sociedad Geográfica-Digital Earth (RSG-DE CoE), en el que también participan diversas universidades españolas como la Universidad Complutense de Madrid y la de León, así como otros profesores de universidad y de enseñanzas no universitarias, preocupados por esta línea innovadora de enseñanza y aprendizaje de la Geografía mediante tecnologías emergentes y «geo-media».

${ }^{6}$ González, M. J. y Lázaro, M. L. (2011): "La geoinformación y su importancia para las tecnologías de la información geográfica”.Ar@cne. Revista electrónica de recursos en Internet sobre Geografía y Ciencias Sociales, 148/1 de junio de 2011. Disponbible en: http://www.ub.es/geocrit/aracne/aracne-148.htm (Fecha de consulta: 01/08/2013). 


\section{EL CRECIMIENTO DE LA ASOCIACIÓN Y SU VOCACIÓN INVESTIGADORA}

La difusión de la Asociación ha ido creciendo, no sólo por el número de participantes, sino por su creciente presencia en las redes sociales con más de dos millares de personas conectadas a través de LinkedIn y varios centenares a través de Faceboook y Twitter.

La participación de la Asociación se ha extendido, no sólo hacia las citadas conferencias de asociaciones científicas internacionales (Unión Geográfica Internacional, EUGEO), sino también a aquellas otras relacionadas con la enseñanza y aprendizaje de la Geografía, como son las de la Geographical Association, del Reino Unido, o las regionales de ESRI Europa y América, y otras conferencias regionales o nacionales en Turquía, Chipre, España, ...

EUROGEO ha impulsado la iniciativa Geocube www.geo-cube.eu, para la promoción de la Geografía en Europa, a través de uno de los pilares de HERODOT, coordinado por la profesora de la Universidad de Malta, María Attard ${ }^{7}$. Consiste en un Cubo de Rubik, en el que cada cuadradito de una cara recoge un tema de interés geográfico. La Real Sociedad Geográfica participó activamente en su confección, junto con otras muchas asociaciones y personas. La página ha recibido más de mil visitas y ha sido traducida a varios idiomas. La traducción al español fue realizada por la profesora María del Carmen Mínguez García.

Las actividades de EUROGEO están cada vez más vinculadas a proyectos de investigación, innovación y tecnología, con un enfoque preferente, aunque no único, hacia la búsqueda de una mayor competitividad de la geografía, mediante el aprendizaje basado en la tecnología del siglo xxi y el trabajo en red. Entre los proyectos en los que interviene, que están ahora mismo activos, podemos citar: iGuess 2 project, para la creación y puesta en marcha de un curso para profesores sobre Sistemas de Información Geográfica; SPACIT Project, para la formación del profesorado en temas sobre ciudadanía y pensamiento espacial; PiriReis Project, para la creación de una Asociación de Geógrafos en Turquía; I-USE (Statistics in Education) Project, para el empleo de las estadísticas en Educación... y otros proyectos que se han solicitado posteriormente y están pendientes de evaluación por la Comisión Europea.

Como se puede observar, tanto en los proyectos, como en las conferencias anuales, la asociación cuenta con un amplio y creciente contacto con universidades dedicadas a la Geografía, por lo que podemos decir que ha ido

${ }^{7}$ Attard, María (2010): "Thematic networks as toolboxes: The case of the HERODOT Network for Geography in Europe”. Documents d'anàlisi geogràfica, 56/2, pp. 325-337. 
dando un giro cada vez más académico. La investigación es un elemento cada vez más importante entre sus acciones, lo que queda patente en sus propios estatutos y en el impulso, desde el año 2009, de una revista digital propia, European Journal of Geography (EJG), que se puede consultar en: http://www.eurogeographyjournal.eu/. La revista supone una referencia obligada no sólo para la educación superior de la Geografía Europea, sino para la investigación. Publica artículos originales, innovadores y de calidad que reflejan aspectos teóricos y aplicados de investigación, de aprendizaje y de enseñanza de la Geografía, y promociona esta ciencia como una disciplina, potenciando además la dimensión europea de la misma.

\section{LAS ASOCIACIONES ESPAÑOLAS EN EUROGEO}

No podemos terminar de hablar de EUROGEO sin una referencia obligada a la participación española en la Asociación. Destacamos a la Real Sociedad Geográfica (RSG), que es la asociación española que más activamente ha participado en EUROGEO siguiendo su línea de participación desde el origen en aquellas organizaciones geográficas internacionales que responden a sus fines (Unión Geográfica Internacional, EUGEO, etc.). Su presencia en EUROGEO ha sido siempre institucional colaborando activamente en las redes europeas en las que EUROGEO ha participado. En las memorias anuales de la Real Sociedad Geográfica ${ }^{8}$ publicadas en su propio Boletín, se puede seguir con detalle la actividad internacional de la misma dentro de la Asociación EUROGEO y también de la UGI y EUGEO.

Entre algunas de las actividades en las que ha colaborado la RSG, podemos destacar la organización, junto con la UCM, de la reunión de expertos en SIG del 29 de mayo al 1 de junio del $2008^{9}$. En ella HERODOT agradeció de forma institucional la hospitalidad de España, no sólo a la Real Sociedad Geográfica, sino también a la Exma. Sra. Decana de la Facultad de Geografía e Historia, Dra. D. ${ }^{a}$ Mercedes Molina y las palabras inaugurales al evento impartidas por el entonces Director del Departamento de Geografía Humana, Dr. D. Miguel

${ }^{8}$ El último de estos informes ha sido publicado en las páginas 392-393, del Boletín de la Real Sociedad Geográfica CXLVIII (2012). Disponible en: http://www.realsociedadgeo grafica.com/es/site/ver_boletines.asp (Fecha de consulta. 01/08/2013).

${ }^{9}$ La información sobre el evento se encuentra disponible en: http://www.herodot.net/previous-events.html y los informes elaborados en ella se pueden consultar en: http://www.herodot. net/geography-benchmark.html 
Angel Troitiño. En la reunión se elaboraron dos documentos sobre la enseñanza de los Sistemas de Información Geográfica uno para cada nivel educativo: uno para el Universitario y otro para las Enseñanzas no Universitarias. Estos documentos fueron consensuados en conferencias posteriores y en la reunión de la Red temática celebrada en Salzburgo (Austria), para ser finalmente publicados en el libro de actas de la conferencia anual celebrada en Liverpool (Reino Unido) en septiembre de 2008. Esta obra ha sido depositada en la Biblioteca de la Facultad de Geografía e Historia de la UCM para su consulta $^{10}$.

También podemos destacar la actividad del Grupo de Didáctica de la Geografía de la Asociación de Geógrafos Españoles en EUROGEO y en la red digital-earth.eu, con la celebración del Congreso organizado por la Universidad de Zaragoza en noviembre de 2012, con el nombre de I Congreso Europeo de Didáctica de la Geografía ${ }^{11}$, por ser el primer congreso que el Grupo de Didáctica de la Geografía organiza en colaboración con una asociación europea, EUROGEO, y una red Comenius, digital-earth.eu. El Grupo de Didáctica de la Geografía celebra cada año un congreso, un año toma el nombre de Nacional y otro de Ibérico, por organizarse el primero en el propio seno de la Asociación y en el segundo caso, en colaboración con la Asociación de Profesores de Geografía de Portugal (APG).

Existen otras muchas actividades celebradas en España en colaboración con EUROGEO y las redes europeas que la Asociación impulsa. Su pormenorizada enumeración escapa a estas páginas. Queda por apuntar que miembros de todas estas asociaciones españolas, y de algunas otras, participan de forma individual en los congresos anuales de EUROGEO, que acoge al menos 17 personas asociadas de diversas Comunidades Autónomas (Cataluña, Extremadura, Madrid, León, Zaragoza...), procedentes de diversas universidades españolas.

${ }^{10}$ Donert, Karl y Wall, Glenda (eds.) (2008): Future Prospects in Geography. HERODOT Conference Proceedings, 4-7 september 2008, Liverpool. Liverpool, Hope University, 518 pp.

${ }^{11}$ El propio organizador del Congreso, el Dr. D. Rafael de Miguel González, realizó una amplia y completa reseña sobre lo acontecido en el mismo, en la revista del Grupo de Didáctica de la Geografía (AGE) titulado: "I Congreso Europeo de Didáctica de la Geografía" (Didáctica Geográfica, 13, pp. 151-154). Disponible en: http://www.didacticageografica.es/index.php/didacticageografica/article/view/104/103 (Fecha de consulta: 01/08/2013). 


\section{CONCLUSIONES}

EUROGEO lleva más de 35 años contribuyendo al avance de la Geografía en Europa y sigue trabajando en ello, si bien con un enfoque que se adapta a la evolución tecnológica de la sociedad, prestando cada vez mayor atención a las tecnologías geoespaciales derivadas del impacto del creciente número de servicios basados en la geolocalización. Actualmente, sigue trabajando en la mejora de la formación del profesorado a partir de iniciativas innovadoras (como la red de centros de excelencia), en las últimas tecnologías (en la línea de la Agenda Digital Europea 2020) y en la competitividad derivada del empleo de todas estas herramientas. El aunar todo esto ha obligado a la asociación a prestar un interés creciente a la investigación.

La diversificación de la Geografía en todas sus dimensiones: investigadora, innovadora, formativa, tecnológica, competitiva..., ha hecho que la Asociación no sólo responda a su vocación internacional, sino que la realidad hace que no exista ninguna otra asociación europea con esta marcada «multidimensionalidad» científica, investigadora, académica y cultural.

María Luisa de Lázaro y Torres Universidad Complutense de Madrid 